\title{
Wearable laser Doppler flowmetry for the analysis of microcirculatory changes during intravenous infusion in patients with diabetes mellitus
}

Zharkikh, Elena, Loktionova, Yulia, Kozlov, Igor, Zherebtsova, Angelina, Sidorov, Victor, et al.

Elena V. Zharkikh, Yulia I. Loktionova, Igor O. Kozlov, Angelina I. Zherebtsova, Victor V. Sidorov, Evgeny A. Zherebtsov, Andrey V. Dunaev, Edik U. Rafailov, "Wearable laser Doppler flowmetry for the analysis of microcirculatory changes during intravenous infusion in patients with diabetes mellitus," Proc. SPIE 11363, Tissue Optics and Photonics, 113631K (2 April 2020); doi: $10.1117 / 12.2552464$

SPIE. Event: SPIE Photonics Europe, 2020, Online Only, France 


\title{
Wearable laser Doppler flowmetry for the analysis of microcirculatory changes during intravenous infusion in patients with diabetes mellitus
}

\author{
Elena V. Zharkikha, Yulia I. Loktionova ${ }^{a}$, Igor O. Kozlov ${ }^{a}$, Angelina I. Zherebtsova ${ }^{a}$, Victor V. \\ Sidorov $^{\mathrm{b}}$, Evgeny A. Zherebtsov ${ }^{\mathrm{c}}$, Andrey V. Dunaev ${ }^{\mathrm{a}}$, and Edik U. Rafailov ${ }^{\mathrm{d}}$ \\ ${ }^{a}$ Research Development Center of Biomedical Photonics, Orel State University named after \\ I.S. Turgenev, 95 Komsomolskaya St, Orel, 302026, Russia \\ "SPE "LAZMA", Moscow, 125252, Russia \\ 'Opto-Electronics and Measurement Techniques Research Unit, University of Oulu, Oulu, \\ 90570, Finland \\ ${ }^{\mathrm{d}}$ Aston Institute of Photonic Technologies, Aston University, Aston Triangle, Birmingham, B4 \\ $7 \mathrm{ET}, \mathrm{UK}$
}

\begin{abstract}
The article is aimed at conducting pilot studies of microcirculation changes in patients with diabetes mellitus during the course of intravenous infusions of alpha lipoic acid. The study was conducted with patients with diabetes during the passage of a standard course of treatment. The change in parameters was compared before the start of the infusion course, during and after the course. A system of wearable laser Doppler monitors was used to conduct the study. The study showed that although the microcirculation index does not undergo significant changes during the treatment process, spectral analysis of the recorded signal has potential applicability. Further studies with a larger sample group are required for a qualitative analysis of the observed effects.
\end{abstract}

Keywords: diabetic neuropathy, skin blood flow, alpha lipoic acid, laser Doppler flowmetry, wearable electronics

\section{INTRODUCTION}

Diabetic polyneuropathy (DPN) is one of the common complications of diabetes mellitus (DM), and is also a leading cause of non-traumatic limb amputation. DM is one of the main causes of neuropathy around the world, and in particular in industrialized countries. ${ }^{1}$ Complication is characterized by a complex pathogenetic network of interconnected metabolic, neurotrophic and vascular defects that cause chronic progressive damage and loss of non-myelinated and myelinated peripheral nerve fibers. ${ }^{2}$ The growing incidence of DM raises its complications to the level of the most important problems of modern healthcare. There is a need for new strategies for the effective treatment of DM complications and the prevention of their development. In earlier studies, it was shown that the duration and quality of life of patients with DM largely depend on the presence and degree of chronic complications. ${ }^{3}$

There are several types of diabetic neuropathy, which differ in their manifestations and pathogenesis. ${ }^{2}$ As a result, various tactics of treating this complication are proposed. Since oxidative stress plays a large role in the development of diabetic complications, some studies have shown the beneficial effects of antioxidant therapy on preventing or slowing the development of DPN. Alpha lipoic acid (ALA) is one such substance. It belongs to a number of strong antioxidants, and also has insulin-like effects. ALA has historically been considered an excellent antioxidant and oxidative stress neutralizer. ${ }^{4}$ It has been reported that ALA has a number of potentially beneficial effects both for the prevention and treatment of diseases associated with peroxidation. Among the positive effects of ALA is the fact that it can lower levels of C-reactive protein. ${ }^{5}$

Send correspondence to Elena Zharkikh

E-mail: e.zharkikh@oreluniver.ru, Telephone: +79534740686

Tissue Optics and Photonics, edited by Valery V. Tuchin, Walter C. P. M. Blondel,

Zeev Zalevsky, Proc. of SPIE Vol. 11363, 113631K · (C) 2020 SPIE

CCC code: $0277-786 \mathrm{X} / 20 / \$ 21 \cdot$ doi: $10.1117 / 12.2552464$

Proc. of SPIE Vol. $11363113631 \mathrm{~K}-1$ 
Clinical trials of the substance showed that ALA significantly improves the symptoms of neuropathy, while its intravenous administration is more effective than oral administration. ${ }^{6}$ The best results of therapy are observed when applying a course of intravenous infusions followed by taking the substance orally.

Studies show that the development of DPN is associated not only with metabolic, but also with vascular disorders, and in particular with a decrease in nerve blood flow. ${ }^{7,8}$ Although there have been numerous studies of changes in symptoms of DPN in the treatment of ALA, studies of the effect of this substance on the state of microcirculation are limited. ${ }^{9}$

The aim of this study was to evaluate the change in blood microcirculation parameters during intravenous infusions of a solution of ALA in patients with type 2 DM using a system of wearable laser Doppler monitors.

\section{MATERIALS AND METHODS}

Experimental studies were carried out using four wearable laser Doppler flowmetry monitors "AMT-LAZMA 1" (Aston Medical Technology Ltd., UK) for the analysis of blood microcirculation. ${ }^{10}$ These devices implement the method of laser Doppler flowmetry (LDF) with simultaneous registration of data from the accelerometer and skin thermometer. As the emitter in the sensor is used in a vertical-cavity surface-emitting laser (VCSEL) with a wavelength of $850 \mathrm{~nm}$, the signal receivers are two photodiodes.

The LDF method is based on the light probing of the tissue and subsequent analysis of reflected from moving red blood cells light. LDF is used for functional diagnostics of the blood microcirculation system, including diagnostics of socially significant diseases associated with the cardiovascular system. ${ }^{11}$ In addition, this method allows one to evaluate oscillatory processes in the microcirculatory bed using data processing by the means of the Morlet wavelet. There are several frequency ranges that characterize the contribution of various factors to the LDF signal: endothelial $(0.0095-0.021 \mathrm{~Hz})$, neurogenic $(0.021-0.052 \mathrm{~Hz})$, myogenic $(0.052-0.145 \mathrm{~Hz})$, respiratory $(0.145-0.6 \mathrm{~Hz})$, and cardiac $(0.6-2 \mathrm{~Hz}){ }^{12}$ Analysis of skin blood flow fluctuations significantly aids understanding the physiological characteristics of the cardiovascular system, ${ }^{13}$ and allows one to track pathological changes in various diseases, in particular in rheumatic diseases ${ }^{11}$ and diabetes. ${ }^{14,15}$

The research was carried out according to the following scheme shown in the figure 1 . Infusion procedures for patients start from the 2nd day of the patient's stay in the hospital. Three studies were performed on the first infusion day using the following algorithm: 10 minutes of recording basal blood flow before infusion, about 20 minutes of recording blood flow during infusion (the recording time may differ slightly between patients), 10 minutes of recording basal blood flow immediately after the infusion procedure. On the second day, a 10-minute recording of basal blood flow was performed before the second infusion procedure. The last 10-minute recording of basal blood flow was made on the day after the end of the infusion procedures that is, on the 6th day of the patient's stay in the hospital (each patient underwent a course of 5 infusions).

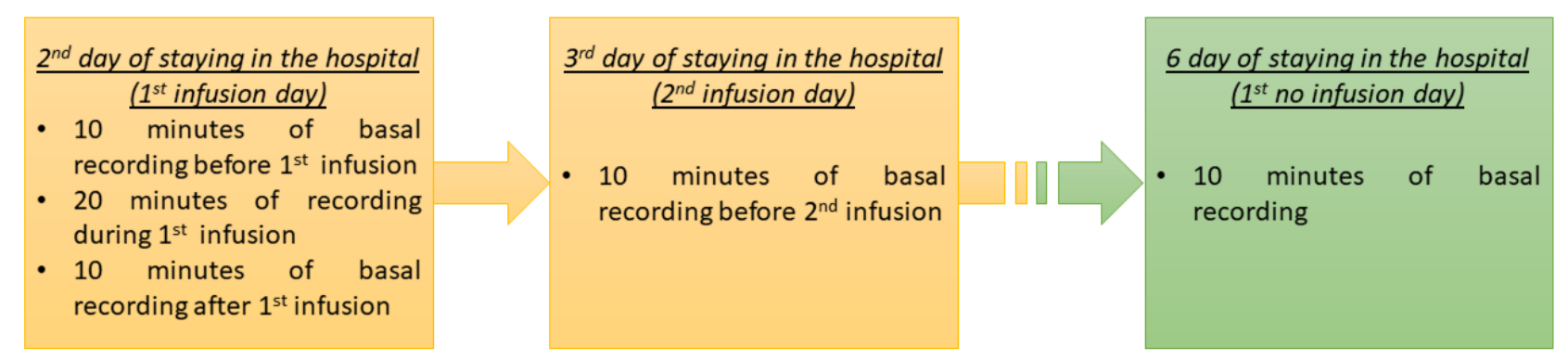

Figure 1. The protocol of the experimental studies

During all recordings, wearable laser analyzers were attached to the plantar side of the big toes and the palmar surface of the distal phalanx of the 3rd fingers. The patients rested supine. The ALA solution was infused into the lateral subcutaneous vein of the right or left hand. The choice of hand was determined by the medical personnel. The study involved 10 patients with type 2 diabetes mellitus ( 4 men and 6 women, average age $55.8 \pm 14.3)$. 
To detect the body's response to the course of ALA infusions, the microcirculation index $\left(I_{m}\right)$, the amplitudes of endothelial $(E)$, neurogenic $(N)$, myogenic $(M)$, respiratory $(R)$ and cardiac $(C)$ oscillations, normalized for standard deviation, were evaluated. To assess the impact of intravenous infusions on the signal registered, all analyzed parameters were normalized to the data of the first stage (baseline).

\section{RESULTS AND DISCUSSION}

In this study, long-term monitoring of the microhaemodynamic parameters was carried out during a standard course of treatment for patients with DM. During the research, the initial values of the measured parameters were recorded at the time the patient was admitted to the hospital before any therapeutic effects were exerted.
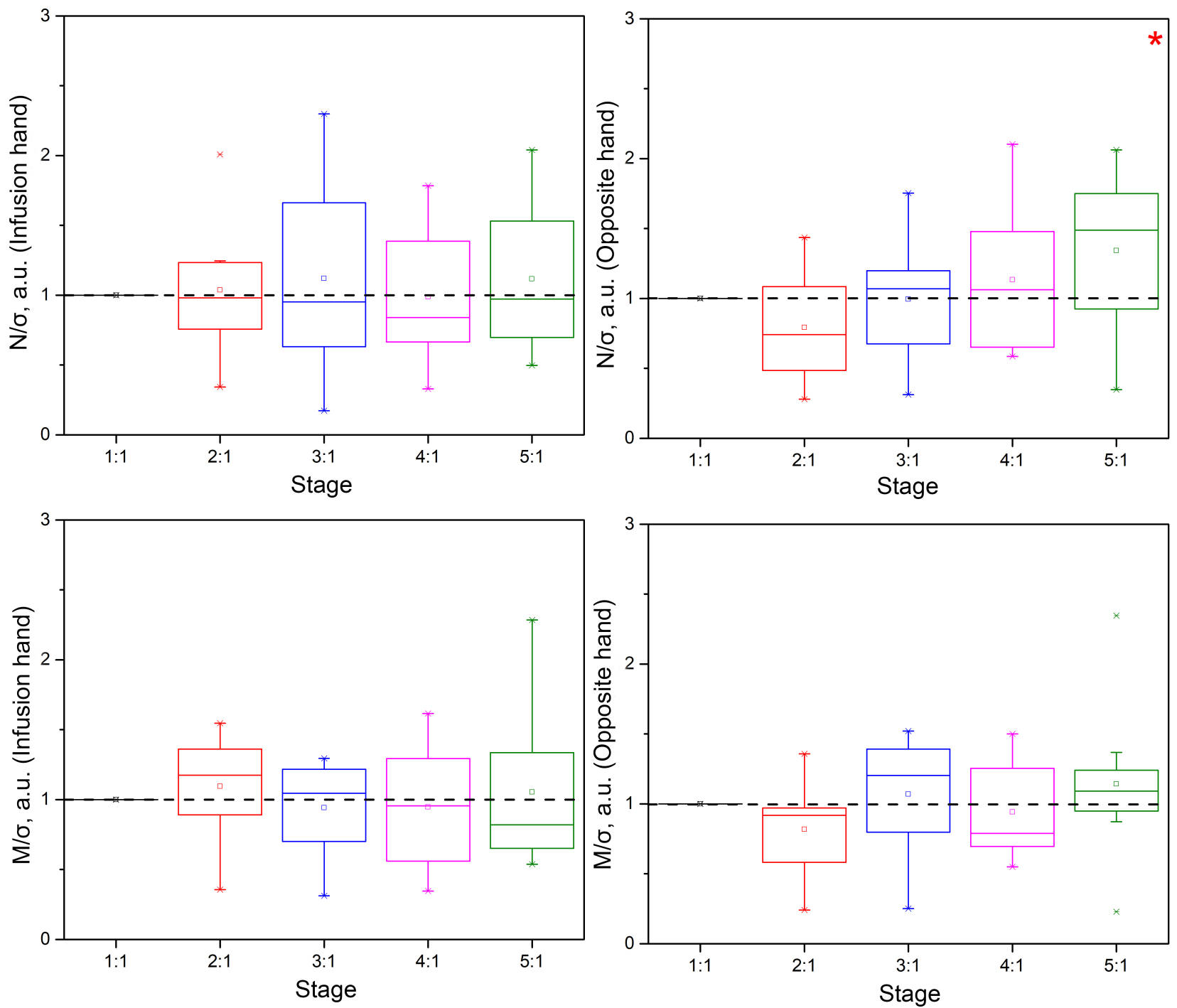

Figure 2. Relative increment of neurogenic (upper panel) and myogenic (lower panel) oscillations measured in the fingers * - The significance of the difference between the values was confirmed with $p<0.05$ according to the Friedman-Anova test

The index of microcirculation, measured in all 4 areas of the study, showed a tendency to decrease by the time the therapy is completed. Although there were no statistically significant differences between the measurements taken before and after therapy, a trend for a slight decrease in microcirculation was observed in all studied 
patients. Taking into account the fact that patients with diabetes are often associated with increased values of the index of microcirculation in comparison with a healthy controls, ${ }^{14,15}$ this change can be assumed due to the positive effects of the therapy provided.

It is interesting to note that during the treatment process significant changes in neurogenic fluctuations are observed, while the reaction varies for the upper and lower extremities. By the last stage, an increase in normalized oscillations in the hands is observed, while in the legs there is a trend towards a decrease in this parameter. Oscillations of the myogenic range, although underwent growth by the time of therapy completion, did not reach statistically significant differences compared with the first examination. In myogenic oscillations, different changes were observed in the upper and lower extremities, as indicated above for neurogenic ones. Figure 2 shows the relative increment of neurogenic (upper panel) and myogenic (lower panel) oscillations measured in the fingers.

It is worth noting that the absence of significant changes in the measured parameters can be correlated with the short duration of the course of treatment. Indeed, it was noted that a significant improvement in the symptoms of neuropathy occurs after an 8-week course of treatment using ALA. ${ }^{5}$ In addition, treatment with ALA has a cumulative effect and may not appear in the microcirculation parameters during the first week. In medical practice, mixed courses with consumption of ALA in the form of pills for several weeks after a course of intravenous infusion are more common and show better results. A promising direction is to study the long-term effect of ALA intake on the state of microcirculation in patients after undergoing an intravenous infusion course, which will be the subject of our further studies.

\section{CONCLUSION}

The presented study demonstrates the possibility of using the LDF method to assess changes in haemodynamic parameters in the treatment of diabetes complications. It was shown that wearable LDF devices are capable of the estimating of the microcirculatory parameters during intravenous infusions, as well as of tracing the dynamic changes in parameters during treatment.

In the present study, it was shown that the use of ALA contributes to a change in the regulation parameters of microcirculatory blood flow, in particular, fluctuations of the neurogenic and myogenic origin. However, these observations require further research with a larger sample size and longer monitoring. The research technique can be applied not only to ALA infusions, but also to study another potential treatment option.

The use of functional tests to assess the reactivity of the microcirculatory system, such as the occlusion test, local tissue heating, etc., has potential opportunities for a better assessment of the effectiveness of therapy. The use of functional tests allowing to evaluate the state of microcirculation in dynamics and study its adaptive properties, which may be impaired as a result of the disease.

Finally, since ALA is an antioxidant and is used to treat metabolic disorders, a multimodal approach using other biophotonic methods simultaneously in the same diagnostic volume seems to be preferable.

\section{ACKNOWLEDGMENTS}

This study was funded by the Russian Science Foundation (the research project 18-79-00237).

\section{REFERENCES}

[1] Edwards, J. L., Vincent, A. M., Cheng, H. T., and Feldman, E. L., "Diabetic neuropathy: mechanisms to management," Pharmacology \& therapeutics 120(1), 1-34 (2008).

[2] Said, G., "Diabetic neuropathy - a review," Nature clinical practice Neurology 3(6), 331-340 (2007).

[3] Wexler, D., Grant, R., Wittenberg, E., Bosch, J., Cagliero, E., Delahanty, L., Blais, M., and Meigs, J., "Correlates of health-related quality of life in type 2 diabetes," Diabetologia 49(7), 1489-1497 (2006).

[4] Bjørklund, G., Aaseth, J., Crisponi, G., Rahman, M. M., and Chirumbolo, S., "Insights on alpha lipoic and dihydrolipoic acids as promising scavengers of oxidative stress and possible chelators in mercury toxicology," Journal of inorganic biochemistry 195, 111-119 (2019). 
[5] Saboori, S., Falahi, E., Eslampour, E., Khosroshahi, M. Z., and Rad, E. Y., "Effects of alpha-lipoic acid supplementation on c-reactive protein level: A systematic review and meta-analysis of randomized controlled clinical trials," Nutrition, Metabolism and Cardiovascular Diseases 28(8), 779-786 (2018).

[6] Han, T., Bai, J., Liu, W., and Hu, Y., "A systematic review and meta-analysis of alpha-lipoic acid in the treatment of diabetic peripheral neuropathy," European Journal of Endocrinology 167, 465-471 (2012).

[7] Tesfaye, S., Malik, R., and Ward, J., "Vascular factors in diabetic neuropathy," Diabetologia 37(9), 847-854 (1994).

[8] Cameron, N. E. and Cotter, M. A., "Metabolic and vascular factors in the pathogenesis of diabetic neuropathy," Diabetes 46, S31-S37 (1997).

[9] Jin, H., Joung, S., Park, J., Baek, H., and Park, T., "The effect of $\alpha$-lipoic acid on symptoms and skin blood flow in diabetic neuropathy," Diabetic medicine 24(9), 1034-1038 (2007).

[10] Zherebtsov, E. A., Zharkikh, E. V., Kozlov, I., Zherebtsova, A. I., Loktionova, Y. I., Chichkov, N. B., Rafailov, I. E., Sidorov, V. V., Sokolovski, S. G., Dunaev, A. V., et al., "Novel wearable vcsel-based sensors for multipoint measurements of blood perfusion," Proc. SPIE 10877, 1087708 (2019).

[11] Zherebtsova, A. I., Dremin, V., Makovik, I., Zherebtsov, E. A., Dunaev, A., Goltsov, A., Sokolovski, S. G., and Rafailov, E., "Multimodal optical diagnostics of the microhaemodynamics in upper and lower limbs," Frontiers in physiology 10, 416 (2019).

[12] Lancaster, G., Stefanovska, A., Pesce, M., Vezzoni, G., Loggini, B., Pingitore, R., Ghiara, F., Barachini, P., Cervadoro, G., Romanelli, M., and Rossi, M., "Dynamic markers based on blood perfusion fluctuations for selecting skin melanocytic lesions for biopsy," Scientific Reports 5, 12825 (2015).

[13] Dremin, V., Kozlov, I., Volkov, M., Margaryants, N., Potemkin, A., Zherebtsov, E., Dunaev, A., and Gurov, I., "Dynamic evaluation of blood flow microcirculation by combined use of the laser doppler flowmetry and high-speed videocapillaroscopy methods," Journal of Biophotonics 12, e201800317 (2019).

[14] Mizeva, I., Zharkikh, E., Dremin, V., Zherebtsov, E., Makovik, I., Potapova, E., and Dunaev, A., "Spectral analysis of the blood flow in the foot microvascular bed during thermal testing in patients with diabetes mellitus," Microvascular research 120, 13-20 (2018).

[15] Zherebtsov, E. A., Zharkikh, E. V., Kozlov, I. O., Loktionova, Y. I., Zherebtsova, A. I., Rafailov, I. E., Sokolovski, S. G., Sidorov, V. V., Dunaev, A. V., and Rafailov, E. U., "Wearable sensor system for multipoint measurements of blood perfusion: pilot studies in patients with diabetes mellitus," Proc. SPIE 11079, 1107962 (2019). 